\title{
Characterization of novel microsatellite markers derived from Korean rose bitterling (Rhodeus uyekii) genomic library
}

\author{
W.J. Kim ${ }^{1}$, E.H. Shin ${ }^{1}$, H.J. Kong ${ }^{1}$, H.S. Kim ${ }^{1}$, B.S. Kim ${ }^{1}$, B.H. Nam ${ }^{1}$, \\ Y.O. Kim ${ }^{1}$, C.H. Kim ${ }^{2}$, H. Jung ${ }^{1}$ and C.M. An ${ }^{1}$ \\ ${ }^{1}$ Biotechnology Research Division, \\ National Fisheries Research and Development Institute, Busan, \\ Republic of Korea \\ ${ }^{2}$ Central Regional Inland Fisheries Research, \\ National Fisheries Research and Development Institute, \\ Gyeonggi-do, Republic of Korea \\ Corresponding author: W.J. Kim \\ E-mail:wj2464@korea.kr
}

Genet. Mol. Res. 13 (4): 8147-8152 (2014)

Received August 9, 2013

Accepted May 29, 2014

Published October 7, 2014

DOI http://dx.doi.org/10.4238/2014.October.7.9

\begin{abstract}
Korean rose bitterling (Rhodeus uyekii) is a freshwater fish endemic to Korea. Natural populations of this species have experienced severe declines as a result of habitat fragmentation and water pollution. To conserve and restore $R$. uyekii, the genetic diversity of this species needs to be assessed at the population level. Eighteen novel polymorphic microsatellite loci for $R$. uyekii were developed using an enriched partial genomic library. Polymorphisms at these loci were studied in 150 individuals collected from three populations. The number of alleles at each locus ranged from 3 to 47 (mean $=17.1)$. Within the populations, the observed heterozygosity ranged from 0.032 to 1.000 , expected heterozygosity from 0.082 to 0.967 , and polymorphism information content from 0.078 to 0.950 . Six loci showed significant deviation from Hardy-Weinberg equilibrium after Bonferroni's correction, and no
\end{abstract}


significant linkage disequilibrium was detected between most locus pairs, except in three cases. These highly informative microsatellite markers should be useful for genetic population structure analyses of $R$. uyekii.

Key words: Rhodeus uyekii; Korean rose bitterling; Microsatellite loci; Genetic diversity

\section{INTRODUCTION}

Bitterlings belong to the subfamily Acheilognathinae, which includes approximately 40 species and subspecies. They are distributed in temperate regions of Europe and Asia, including Korea, Japan, Taiwan, and China (Banarescu, 1990). Korean bitterlings are classified into 2 genera and 14 species, including 9 endemic species (Kim et al., 2005). The Korean rose bitterling (Rhodeus uyekii) is a common freshwater fish endemic to Korea, and it inhabits tributaries of the Nagdong, North Han, and Tamjin Rivers. Recently, this species was named as a candidate for development as an ornamental fish because of its small size and beautiful coloration (Kang et al., 2005). Natural populations of this species have recently experienced severe declines as a result of overfishing, habitat fragmentation, and water pollution.

Conservation projects are currently under way to promote increases in population size and distribution. However, knowledge of the population genetic structure for this species is essential to develop effective plans, and suitable DNA markers are needed for the evaluation of population genetic diversity. Because microsatellite markers have high numbers of polymorphisms, codominant inheritance, a genome-wide distribution, and high reproducibility, they are the most popular and powerful molecular markers in population genetics research (Liu and Cordes, 2004). In the present study, we developed the first set of microsatellite makers for $R$. uyekii and assessed polymorphisms at these loci in 150 individuals collected from three different populations.

\section{MATERIAL AND METHODS}

Genomic DNA was extracted from muscle tissue of $R$. uyekii using the TNES-urea buffer method (Asahida et al., 1996). A partial genomic library enriched for GT repeats was constructed using a slightly modified version of the procedures described by Hamilton et al. (1999). The DNA was then digested with $A l u \mathrm{I}, R s a \mathrm{I}$, and HaeIII (New England Biolabs, Ipswich, MA, USA), and DNA fragments ranging from 300 to $1000 \mathrm{bp}$ were isolated and ligated to SNX/SNX rev linker sequences. The linker-ligated DNA was amplified by polymerase chain reaction (PCR) using SNX as the primer, and the products were hybridized to biotinylated (GT) $)_{10}$ probes attached to streptavidin-coated magnetic beads (Promega, Madison, WI, USA). The enriched fragments were amplified again; the products were digested with NheI and ligated into the XbaI-digested pUC18 vector (Pharmacia LKB Biotechnology Inc., Gaithersburg, MD, USA), and the resulting constructs were transformed into competent Escherichia coli DH5 $\alpha$ cells. Positive clones with repeats were identified by PCR with (GT) ${ }_{10}$ and M13 primers. A negative control with no template was included in each set of reactions. The PCR products were analyzed on $1.5 \%$ agarose gels, and those clones producing two or more bands were considered to contain a microsatellite locus. Plasmid DNA from the positive clones was purified using AcroPrep 96-well filter plates (Pall Corp., Port Washington, NY, USA). All 
positive colonies were bidirectionally sequenced using M13 forward or reverse primer with a BigDye Terminator Cycle Sequencing Ready Reaction kit and an ABI 3130xl automated DNA sequencer (Applied Biosystems, Foster City, CA, USA). Primers were designed on the basis of the sequences flanking the microsatellite motifs using the Oligo 5.0 software (National Biosciences Inc., Plymouth, MN, USA). The PCR conditions were initially optimized with the DNA samples originally used for microsatellite isolation to establish whether a product of the desired size was amplified by changing the annealing temperature, primers, and $\mathrm{MgCl}_{2}$ concentration. Thirty-two microsatellite loci were genotyped to test the level of genetic polymorphisms using 150 individuals from three natural populations in Cheongpyeong Stream (N =59; CS), Yangcheon Stream ( $=60$; YS), and Tamjin Stream $(\mathrm{N}=31 ; \mathrm{TS})$ in Korea.

Amplification was performed in a $10-\mu \mathrm{L}$ reaction mixture containing $10 \mathrm{ng}$ genomic DNA, 1X PCR buffer, $1.5 \mathrm{mM} \mathrm{MgCl}, 0.2 \mathrm{mM}$ each dNTP, 3 pmol of each primer, $0.5 \mathrm{X}$ Band Doctor, and $0.5 \mathrm{U}$ f-Taq DNA polymerase (Solgent Co. Ltd., Daejeon, Korea). The forward primers were end-labeled commercially with the dyes 6-FAM, NED, or HEX (Applied Biosystems). The reactions were conducted using a PTC-200 thermocycler (MJ Research Inc., Waltham, MA, USA) with an initial denaturation at $95^{\circ} \mathrm{C}$ for $15 \mathrm{~min}$, followed by 35 cycles of $30 \mathrm{~s}$ at $95^{\circ} \mathrm{C}, 30 \mathrm{~s}$ at a primer-specific annealing temperature (Table 1), and $30 \mathrm{~s}$ at $72^{\circ} \mathrm{C}$, with a final $30 \mathrm{~min}$ extension at $72^{\circ} \mathrm{C}$. The lengths of the products were determined with an ABI $3130 x l$ Genetic Analyzer (Applied Biosystems) using the GeneScan-400HD (ROX) size standard (Applied Biosystems).

The number of alleles, polymorphism information content (PIC), and observed and expected heterozygosity at each locus were calculated using Cervus 3.03 (Marshall et al., 1998). Deviations from Hardy-Weinberg equilibrium (HWE) and linkage disequilibrium were estimated using Genepop 4.0 (Raymond and Rousset, 1995), and adjusted P values for both analyses were obtained using a sequential Bonferroni test for multiple comparisons (Rice, 1989). We also estimated inbreeding coefficient $\left(F_{\text {IS }}\right)$ values (Weir and Cockerham, 1984), which can identify departures from HWE within a population. The presence of null alleles was examined using Microchecker 2.2.3 (Van Oosterhout et al., 2004).

\section{RESULTS AND DISCUSSION}

In total, 400 white colonies with inserts were randomly selected and screened for repeats using PCR, yielding $250(62.5 \%)$ true positive clones. These were sequenced, producing a total of $218(54.5 \%)$ sequences containing simple sequence repeats, of which $163(40.8 \%)$ were eliminated because they possessed no flanking sequences. Fifty-five (13.8\%) sequences containing microsatellites were obtained and primers were designed to amplify microsatellitecontaining regions of the genome. Only 32 of the 55 primer pairs tested successfully amplified the target region, and the remaining pairs either failed to amplify or produced nonspecific bands. We labeled 32 primer pairs with fluorescent dyes. When the PCR products were genotyped, 11 were not scorable due to excessive stutter and 3 were monomorphic. Ultimately, we chose 18 microsatellite markers and examined the genetic diversity of 150 individuals collected from three populations. The number of alleles, repeat motif, product size range, optimized annealing temperature, and GenBank accession number for each of the 18 microsatellite loci are presented in Table 1. In total, 308 alleles were detected at the 18 loci analyzed in 150 individuals. The number of alleles per locus varied from 3 to 47 , with a mean of 17.1 in the total population. Within populations, the observed heterozygosity ranged from 0.032 to 
1.000, expected heterozygosity from 0.082 to 0.967 , and PIC from 0.078 to 0.950 (Table 2). Although it varied among loci, the mean observed heterozygosity was lower than the mean expected heterozygosity in all three populations. Significant deviations from HWE after sequential Bonferroni correction $(\mathrm{P}<0.0028)$ were detected at six loci in at least one population [Ru48 (CS), Ru129 (CP and TS), Ru249 (CS), Ru276 (TS), Ru573 (TS), and Ru792 (TS)] (Table 2). A significant heterozygote deficiency was detected at these loci. An analysis with Microchecker indicated the possible occurrence of null alleles at five loci, except Ru792, but no evidence of allelic dropout was found for any of the loci (Bonferroni's correction). These five loci showed a highly significant positive $F_{\text {IS }}$ (i.e., heterozygote deficiency) together with a high estimated null allele frequency, strongly suggesting a causative relationship. In addition, no significant linkage disequilibrium between loci was detected for most of the locus pairs after Bonferroni's correction ( $\mathrm{P}<0.0028)$, except in three cases (Ru48-Ru235, Ru48-Ru280, and Ru235-Ru280) (Table 2). These microsatellite markers will be useful for studying the population genetic structure and establishing effective conservation strategies for $R$. uyekii. We are currently analyzing the fine-scale structural variation in Korean $R$. uyekii populations.

Table 1. Primer sequences and characteristics of 18 polymorphic microsatellite loci in Rhodeus uyekii.

\begin{tabular}{|c|c|c|c|c|c|}
\hline Locus & Repeat motif & Primer sequence $\left(5^{\prime}-3^{\prime}\right)$ & $\mathrm{Ta}\left({ }^{\circ} \mathrm{C}\right)$ & Size range (bp)* & GenBank accession No. \\
\hline Ru35 & $(\mathrm{AC})_{14}$ & $\begin{array}{l}\text { F: CACCGCATGCTTCTTTAATATCCAG } \\
\text { R: ATGTAAAGTCCGCGTGCTTGTG }\end{array}$ & 55 & $160-194$ & KF049471 \\
\hline Ru48 & $(\mathrm{GT})_{13}$ & $\begin{array}{l}\text { F: ACATACGTGCCGATCTTCTTA } \\
\text { R: TGTGTTGATCAGGTGTTCTCA }\end{array}$ & 55 & 109-199 & KF049472 \\
\hline Ru62 & $(\mathrm{GT})_{31}$ & $\begin{array}{l}\text { F: CTCTCACGCTTTCTCCATTC } \\
\text { R: GTAATAGAGTTTTGATTCCCAACC }\end{array}$ & 58 & 203-351 & KF049473 \\
\hline Ru127 & $(\mathrm{GAG})_{9}$ & $\begin{array}{l}\text { F: ATTCACACTCCTGCTTCCTGAC } \\
\text { R: TATGATAGCAAAGAGAACGAAGGG }\end{array}$ & 60 & $207-216$ & KF049474 \\
\hline Ru129 & $(\mathrm{GT})_{11}$ & $\begin{array}{l}\text { F: GAGGAAAACAGAAAGCGATTCATC } \\
\text { R: GACCTCCAGCCCTCATTCAAT }\end{array}$ & 55 & $241-289$ & KF049475 \\
\hline Ru132 & $(\mathrm{AC})_{15} \mathrm{GC}(\mathrm{AC})_{8}$ & $\begin{array}{l}\text { F: CCTGTGCAATTGAGTTTGACAC } \\
\text { R: TGTTTCCTTTCTCGACCTGTTTAC }\end{array}$ & 60 & $117-149$ & KF049476 \\
\hline Ru235 & $(\mathrm{GA})_{10} \mathrm{GG}(\mathrm{GA})_{8}$ & $\begin{array}{l}\text { F: GAAGTAAGCCTCCCACAAGAACTG } \\
\text { R: GCAGCAGGGCACTGATTTATCT }\end{array}$ & 60 & $119-217$ & KF049477 \\
\hline Ru249 & $(\mathrm{GT})_{3}(\mathrm{GAGT})_{3}(\mathrm{GT})_{5}$ & $\begin{array}{l}\text { F: TTGACCGTATGTTGACCTCCTG } \\
\text { R: TTACAGCCAGAAGACCAGCATTTA }\end{array}$ & 60 & $54-236$ & KF049478 \\
\hline Ru276 & $(\mathrm{GT})_{20}$ & $\begin{array}{l}\text { F: ACACGCCTCACCATCATATCACAG } \\
\text { R: CAAACGCTTCACGCCATCAG }\end{array}$ & 55 & $250-274$ & KF049479 \\
\hline Ru280 & $(\mathrm{AC})_{8}$ & $\begin{array}{l}\text { F: CTTGTCCTCTGCATCTCTTTCTCA } \\
\text { R: ACAAATGACACATGCTTCCACTG }\end{array}$ & 58 & $60-72$ & KF049480 \\
\hline Ru382 & $(\mathrm{AC})_{10}$ & $\begin{array}{l}\text { F: TTTCCCATTATACTTCTTACAGCA } \\
\text { R: TATACCTCCCTTTTTATGTCACTG }\end{array}$ & 58 & $128-138$ & KF049481 \\
\hline Ru396 & $(\mathrm{GT})_{11}$ & $\begin{array}{l}\text { F: TGATTCTTCTCTCGGCTGGTTG } \\
\text { R: GAGGTTTACTGATTCAGCGATGGA }\end{array}$ & 60 & $111-153$ & KF049482 \\
\hline Ru414 & $(\mathrm{GT})_{12}$ & $\begin{array}{l}\text { F: TCCATCTGTCCTCCAACACAAAAT } \\
\text { R: CCAACTCCACCTTCCCCTGTAA }\end{array}$ & 55 & $225-263$ & KF049483 \\
\hline Ru461 & $(\mathrm{CT})_{10}$ & $\begin{array}{l}\text { F: ACTGAACTCCCTACTAAGATGATT } \\
\text { R: GGGAGAAAGTGAGAGGTAAAG }\end{array}$ & 58 & $109-141$ & KF049484 \\
\hline Ru573 & $(\mathrm{GT})_{20}$ & $\begin{array}{l}\text { F: TTATGGTTCAGATGTGGCATTATT } \\
\text { R: TGTATGTGACCTCCAGTGCTG }\end{array}$ & 58 & $146-248$ & KF049485 \\
\hline Ru657 & $(\mathrm{AC})_{15}$ & $\begin{array}{l}\text { F: TGCTGACACCTGCTGGACACA } \\
\text { R: GCTCCATTTCTGCAGGAACATTCA }\end{array}$ & 55 & $155-189$ & KF049486 \\
\hline Ru721 & $(\mathrm{GT})_{17} \mathrm{AT}(\mathrm{GT})_{8}$ & $\begin{array}{l}\text { F: ATCAATGAATGAAACAAGTGCTGT } \\
\text { R: TTAGACCATCTGCGAAACTACAAA }\end{array}$ & 60 & $82-240$ & KF049487 \\
\hline Ru792 & $(\mathrm{AC})_{20}$ & $\begin{array}{l}\text { F: TCACATGGCACTTCCTCCGTGTTG } \\
\text { R: CCTGGTTTCCTGTCGGCTCCTGTC }\end{array}$ & 60 & $152-186$ & KF049488 \\
\hline
\end{tabular}

*Size range of alleles genotyped by 150 individuals pooled from three populations. $\mathrm{F}=$ forward primer sequences; $\mathrm{R}=$ reverse primer sequences; $\mathrm{Ta}=$ annealing temperature. 


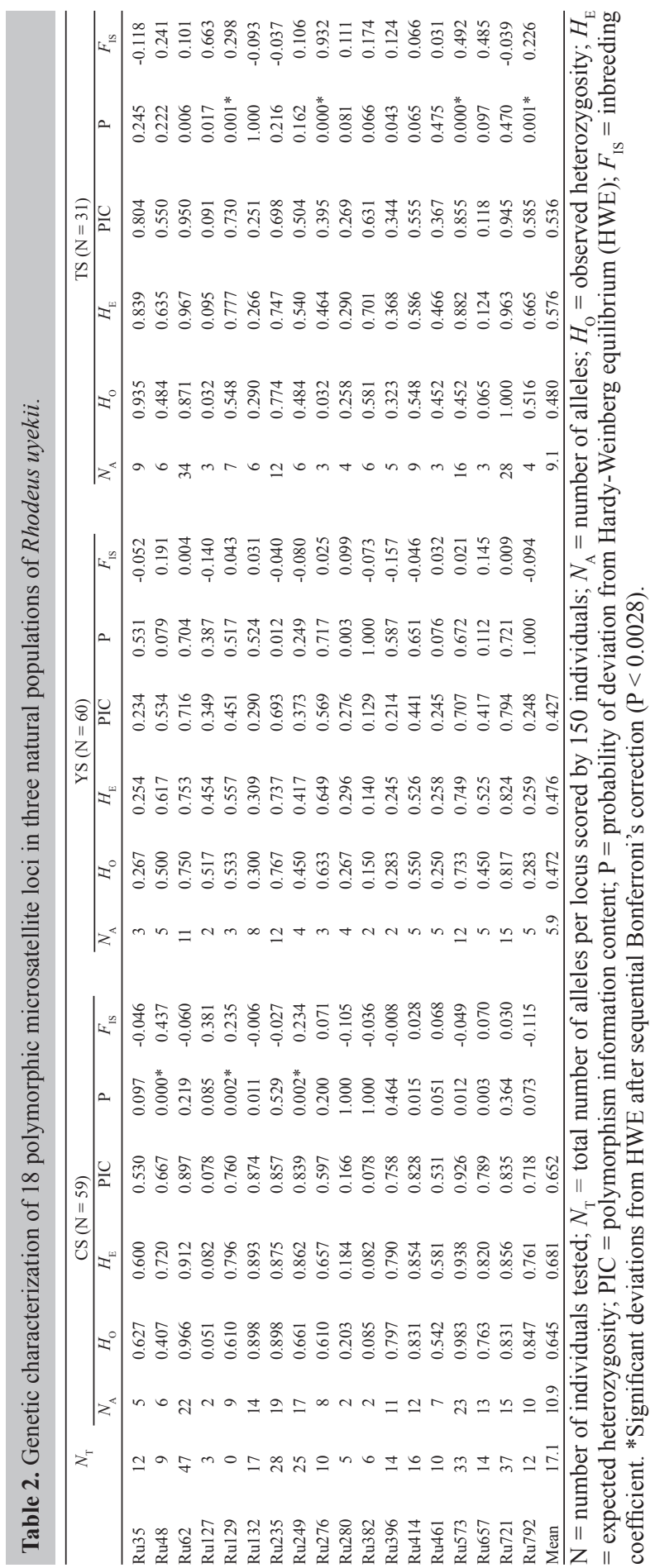




\section{ACKNOWLEDGMENTS}

Research supported by grants from the National Fisheries Research and Development Institute (\#RP-2014-BT-012).

\section{REFERENCES}

Asahida T, Kobayashi T, Saitoh K and Nakayama I (1996). Tissue preservation and total DNA extraction from fish stored at ambient temperature using buffers containing high concentration of urea. Fish. Sci. 62: 727-730.

Banarescu P (1990). Zoogeography of Fresh Waters. General Distribution and Dispersal of Freshwater Animals. AulaVerlag, Wiesbaden.

Hamilton MB, Pincus EL, DiFiore A and Fleischer RC (1999). A universal linker and ligation procedures for construction of genomic DNA libraries enriched for microsatellites. BioTechniques 27: 500-507.

Kang EJ, Kim EM, Kim YJ, Lim SG, et al. (2005). Effect of lidocaine hydrochloride and clove oil as an anaesthetic on Korean rose bitterling, Rhodeus uyekii and oily bitterling, Acheilognathus koreensis. J. Aquac. 18: 272 -279.

Kim IS, Choi Y, Lee CL, Lee YJ, et al. (2005). Illustrated Book of Korean Fishes. Kyu-Hak Publishing Co., Seoul.

Liu ZJ and Cordes JF (2004). DNA marker technologies and their applications in aquaculture genetics. Aquaculture 238: 1-37.

Marshall TC, Slate J, Kruuk LE and Pemberton JM (1998). Statistical confidence for likelihood-based paternity inference in natural populations. Mol. Ecol. 7: 639-655.

Raymond M and Rousset F (1995). GENEPOP (version 1.2): population genetics software for exact tests and ecumenicism. J. Hered. 86: 248-249.

Rice WR (1989). Analyzing tables of statistical tests. Evolution 43: 223-225.

Van Oosterhout CV, Hutchinson WF, Wills DPM and Shipley P (2004). MICRO-CHECKER: software for identifying and correcting genotyping errors in microsatellite data. Mol. Ecol. Notes 4: 535-538.

Weir BS and Cockerham CC (1984). Estimating F-statistics for the analysis of population structure. Evolution 38: 13581370. 